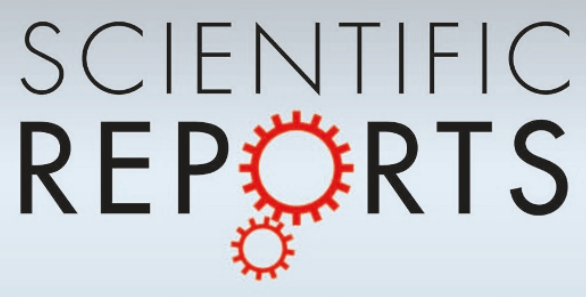

\title{
OPEN Quantum Mechanical Metric for Internal Cohesion in Cement Crystals
}

SUBJECT AREAS:

MATERIALS SCIENCE

THEORY AND COMPUTATION

Received

15 August 2014

Accepted

11 November 2014

Published

5 December 2014

Correspondence and requests for materials should be addressed to W.Y.C. (ChingW@ umkc.edu)

\author{
C. C. Dharmawardhana', A. Misra² \& Wai-Yim Ching'
} 'Department of Physics and Astronomy, University of Missouri - Kansas City, 5110 Rockhill Road, Kansas City, MO 64110, USA,
${ }^{2}$ Department of Civil, Environmental, and Architectural Engineering, University of Kansas, $1530 \mathrm{~W} 15^{\text {th }}$ Street, Lawrence, KS
66045 , USA.

Calcium silicate hydrate (CSH) is the main binding phase of Portland cement, the single most important structural material in use worldwide. Due to the complex structure and chemistry of CSH at various length scales, the focus has progressively turned towards its atomic level comprehension. We study electronic structure and bonding of a large subset of the known CSH minerals. Our results reveal a wide range of contributions from each type of bonding, especially hydrogen bonding, which should enable critical analysis of spectroscopic measurements and construction of realistic C-S-H models. We find the total bond order density (TBOD) as the ideal overall metric for assessing crystal cohesion of these complex materials and should replace conventional measures such as Ca:Si ratio. A rarely known orthorhombic phase Suolunite is found to have higher cohesion (TBOD) in comparison to Jennite and Tobermorite, which are considered the backbone of hydrated Portland cement.

E lectronic structure and inter-atomic bonding characteristics are central to the comprehensive understanding of the atomic-scale structure and properties of calcium silicate hydrates (CSH) that are synthesized as Portland cement hydration products. The emerging interest in atomic structure-property relationships is due to the increasingly significant environmental, social and economic impact of the global Portland cement usage and production, which currently stands at 2 billion metric tons. Current experimental findings based upon a variety of spectroscopic and high-resolution microscopic techniques suggest a poorly crystalline or completely disordered atomic structure of these hydrates in hardened cement paste ${ }^{1}$. Based on these experimental data, a number of structural models have been proposed, either as binary solutions ${ }^{2-4}$ or through theoretical modeling using Jennite and Tobermorite type crystals as starting structures ${ }^{1,5-8}$. These efforts have resulted in a wide range of models for $\mathrm{C}-\mathrm{S}-\mathrm{H}^{9-12}$ that are either based on solution theory or limited atomistic simulations using classical molecular dynamics (MD). A key missing ingredient in these efforts is the understanding of bonding characteristics of both the crystalline CSH as well as the more disordered C-S-H. For brevity and clarity, we will follow the cement chemistry notation of CSH and CS to represent calcium silicate hydrate and anhydrate crystals, respectively, and C-S-H to exemplify the more general structure of calcium silicate hydrate found in hardened cement paste.

Crystalline CSH phases range from almost amorphous to highly ordered crystalline phases. Due to their varying degree of crystallinity and the difficulties associated with isolation of pure phases, many uncertainties remain about the composition and structure even for $\mathrm{CSH}$ phases of high crystallinity. Furthermore, due to the complexity of CSH structures, quantitative bonding information, especially $\mathrm{H}$-bonding (HB), are not accessible purely by experimental means without the aid of robust atomistic model for proper interpretation, giving rise to the proverbial chicken-egg problem. In the light of this critical gap in knowledge, it is of paramount importance to investigate a wide variety of crystalline $\mathrm{CSH}$ phases with well-documented crystal structures. Such studies will provide an in-depth insight on the variations in bonding characteristics in relation to the composition and structures of these materials. Without this information, the atomistic models of C-S-H proposed ${ }^{13}$ in the literature will remain speculative.

The uncertainty with respect to position of " $\mathrm{H}$ " was discussed previously in a study of four crystalline CSH crystals $^{14}$. Since HB has a significant presence in CSH, it plays a crucial role in the behavior of these materials. This fact has been generally recognized but rarely studied quantitatively. Clearly, even for crystalline phases, the bonding information cannot be determined purely from experimental data in the absence of refined structures of these phases. Here we take a materials genomic approach and theoretically investigate a broad range of CSH crystals using accurate first principles methods. Out of the 30 crystalline phases listed by Richardson in the 2008 review article ${ }^{1}$, we choose $15 \mathrm{CSH}$ crystals with well-documented structural information, their four possible 
anhydrous precursors, and portlandite. We begin with fully optimized atomic structures of these crystalline phases. We use a supercell approach to address the issue of partial occupancy at certain sites in some crystals. The electronic structures of the refined structures are then calculated. Subsequently, we quantitatively evaluate the bonding characteristics of these crystals through bond order (overlap population) calculations. We analyze the results following the modified Strunz classification of minerals according to the degree of polymerization of the silicate chains in these crystals. The data obtained from crystalline phases can help in the interpretation of experimental spectroscopic measurements leading to realistic models that are consistent with the both electronic-scale and atomistic-scale behaviors for the C-S-H phase in hardened Portland cement.

Classifications of CSH crystals. A comprehensive review by Richardson et. al. ${ }^{1}$ includes an extensive list of $30 \mathrm{CSH}$ crystal phases that are categorized into six groups following Taylor et al. ${ }^{5}$. However, the $\mathrm{CSH}$ crystals can be more appropriately classified according to the Strunz classification ${ }^{15}$ by considering the silica chain polymerization. The notion of silica chain polymerization also has implications on the products of the complex hydration process $^{16,17}$ and carbonation or biological degradation ${ }^{18,19}$ that occurs in Portland cement paste. It has been speculated that the maturation of cement paste involves increasing polymerization of primarily isolated Si tetrahedra to dimers, trimmers and pentamers, eventually forming a 3-dimensional silicate framework ${ }^{20}$.

Table 1 lists 20 crystal phases with well-documented atomic positions used in this study placed into four groups: a, Clinker and hydroxide phase; $\boldsymbol{b}$, nesosubsilicates; $\boldsymbol{c}$, sorosilicates; and $\boldsymbol{d}$, ionosilicates. Each group in Table 1 is arranged in ascending order of calcium to silicon $(\mathrm{C} / \mathrm{S})$ ratio. The clinker phases (a.1 and a.2) and the Portlandite (a.3) are placed in group a. Portlandite is included in this group since it forms the basis for hydration of cement. The remaining crystals are grouped according to the Strunz scheme. In this scheme, the silicate units at each polymerization step are indicated by $\mathbf{Q}^{\mathbf{n}}$, where the Si tetrahedron is connected to $\mathbf{n}$ other silicate tetrahedra.
For example, an isolated silicate tetrahedron not connected to any other is denoted by $\mathbf{Q}^{0}$. The nesosubsilicates with isolated $\left[\mathrm{SiO}_{4}\right]^{-4}$ groups are placed in group $\boldsymbol{b}$. These $\mathrm{CSH}$ phases possess the lowest degree of polymerization. The sorosilicates with $\left[\mathrm{Si}_{2} \mathrm{O}_{7}\right]^{-6}$ groups in the next polymerization step are placed under group $c$. For these phases, the $\left[\mathrm{Si}_{2} \mathrm{O}_{7}\right]^{-6}$ is connected to two isolated tetrahedra via one bridging-O to form the so-called pyrogroups. Thus, all silicon tetrahedra are $\mathbf{Q}^{1}$. These silicon tetrahedra are linked via nonbridging $\mathrm{O}$, to six-, seven- or even eight fold-coordinated $\mathrm{Ca}$ atoms. A special case of sorosilicate structures are those with mixtures of isolated tetrahedra $\left(\mathbf{Q}^{0}\right)$ and finite triple chains $\left(\mathbf{Q}^{2}\right)$, in which the silicon tetrahedra are connected to 6 and 8 -fold coordinated $\mathrm{Ca}$ atoms. The group $\boldsymbol{d}$ contains the inosilicates. These comprise structures with infinitely long chains with a wide variety of $\mathrm{C} / \mathrm{S}$ ratios, ranging from 0.50 in Nekoite (d.1) to 1.5 in Jennite (d.8). According to the degree of polymerization, the phases in this group could be subdivided into inosilicates with single chains (all tetrahedra are $\mathbf{Q}^{2}$ ), and double chains $\left(2 / 3 \mathbf{Q}^{2}\right.$ and $\left.1 / 3 \mathbf{Q}^{3}\right)$. The representative crystals from each group are illustrated in Figure 1. They are Alite (a.2) for group a; Afwillite (b.1) for group b; Suolunite (c.2), Kilichoanite (c.3) for group c; and Anomalous T11 A (d.2), forshgite (d.7) for group d. Similar images of all 20 crystals are displayed in the supporting information (SI) as Figure S1.

Electronic structures of CSH. Although the atomic structures of the crystals in Table 1 are generally available ${ }^{21}$, inaccuracies exits along with some missing data. Section 1 in SI and Figure S1 give additional information on the fully relaxed and corrected structures to reconcile with the chemical formula where applicable. Table 1 gives a summary of the crystal symmetry, chemical formulas, theoretical density, C/S ratio and calculated electronic band gap. Out of the 20 crystals studied, 4 are anhydrate phases; 2 contain purely isolated water molecules, 5 include both isolated water and hydroxyl groups, and rest have only hydroxyl groups. In CSH, hydroxyl groups are mostly connected to $\mathrm{Si}(\mathrm{Si}-\mathrm{OH})$ but in some instances (b.1, b.2, b.3, c.2, c.4, c.5, d.6 and d.7) they can be connected to $\mathrm{Ca}(\mathrm{Ca}-\mathrm{OH})$ as well. The

Table 1 | Mineral names, crystal symmetry and space group symmetry, chemical formulas, Calculated C/S, density, and band gap (in eV) of the 20 crystals in this study

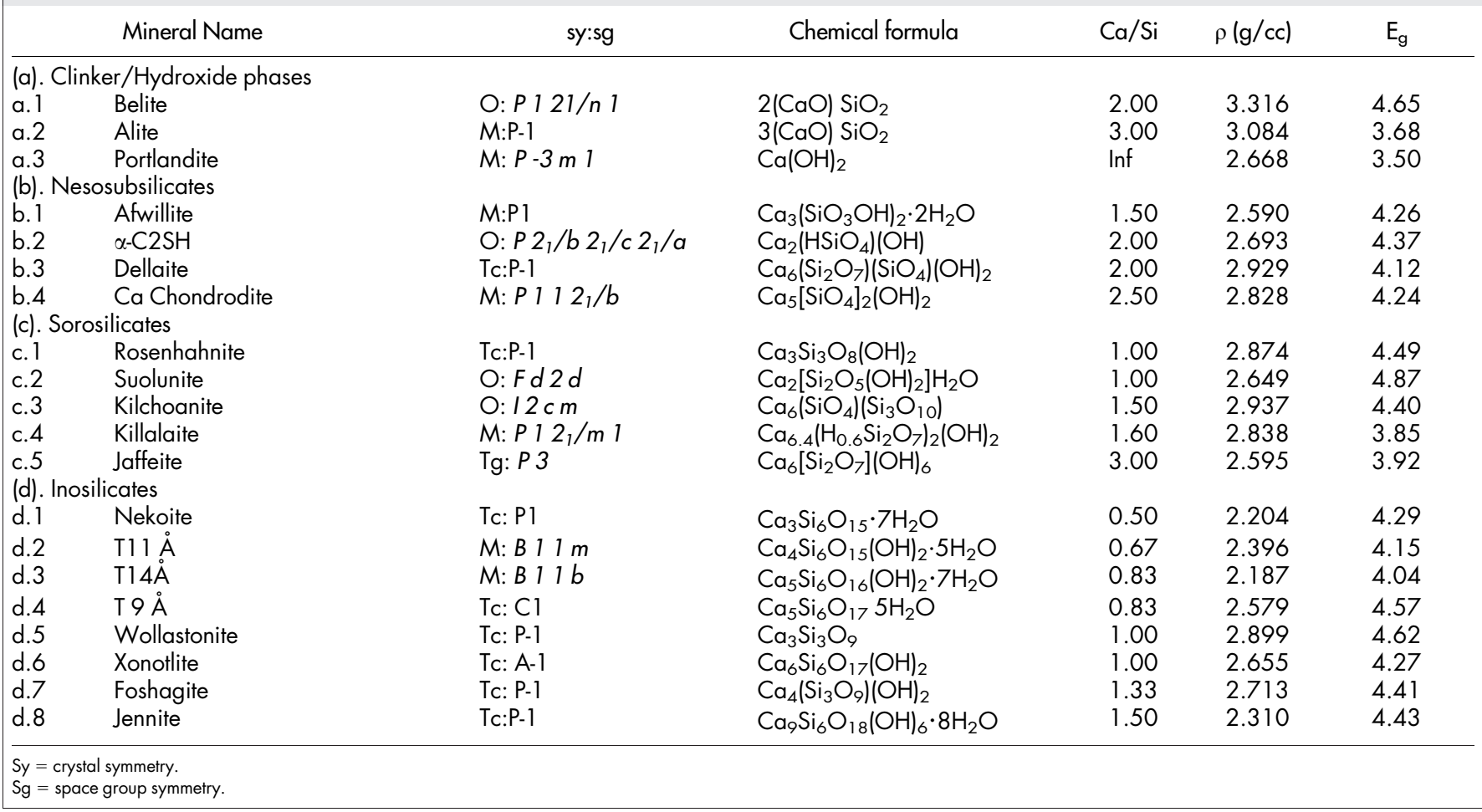


(a.2) Alite

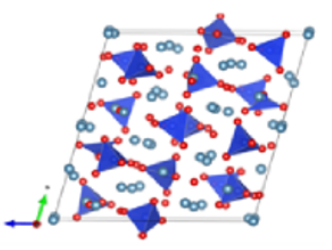

(c.3) Kilchoanite

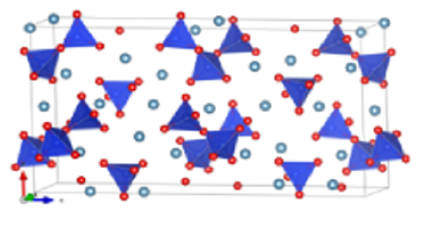

(b.1) Afwillite

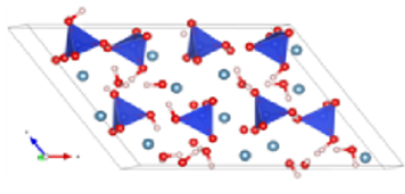

(d.2) Anomalous T11 A

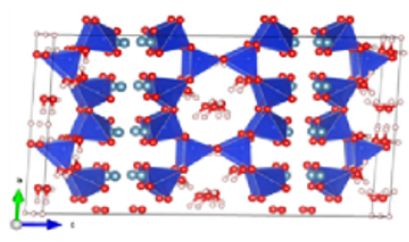

(c.2) Suolunite

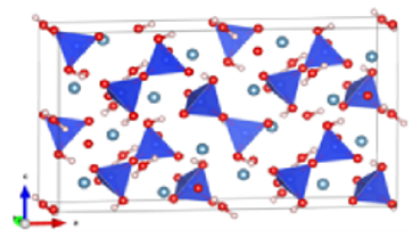

(d.7) Foshagite

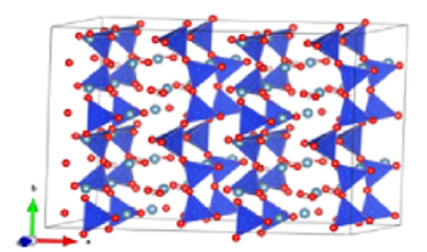

Figure 1 Ball-stick figures of representative crystals to illustrate the structures of each group of CSH and CS crystals: (a.2) Alite; (b.1) Afwillite; (c.2) Suolunite; (c.3) Kilchoanite; (d.2) Anomalous T11 Å; (d.7) Foshagite.

calculated band structures for the 20 crystals are displayed in Figure S2. The zero of the energy is set at the highest occupied state, or the top of the valence band (VB). As expected, all crystals have large band gaps ranging from $3.50 \mathrm{eV}$ to $4.87 \mathrm{eV}$ and exhibit flat top of $\mathrm{VB}$ except a.3. The bottom of the conduction bands (CB) of all crystals have large curvatures suggesting small electron effective mass. Analysis of the partial density of states (PDOS), indicates that the lower part of the VB is from O-2s orbitals and the upper part is from $\mathrm{O}-2 \mathrm{p}$ orbitals of the non-water $\mathrm{O}$ whereas the lower $\mathrm{CB}$ originates mostly from Ca-3d orbitals. There is a significant contribution from $\mathrm{Si}-3 \mathrm{~d}$ orbitals to the upper part of the VB as well. High quality band structure based on accurately relaxed structure is important in assessing the electronic structure because experimentally measured structures are usually not sufficiently accurate. This is particularly true for hydrated crystals with water molecules. Discrepancy with the experimentally assigned " $\mathrm{H}$ " positions was pointed out in earlier work ${ }^{14}$. Table 2 show the VASP relaxed crystal parameters and the percent deviation from the experimentally reported values. The experimental crystal parameters are given in the SI Table S1. The relaxed crystal parameters in general vary $1-2 \%$ compared to the reported experimental values but in some cases such as b.1 and b. 4 , they can be as high as $-4.1 \%$. The highest deviation is in the angle $\gamma$ of the awfillite (b.1) crystal structure. This is a highly hydrated phase and we find considerable deviation in the hydrogen positions from the reported structure. This is explained in more detail in bottom of page 6 . These deviations are larger than those usually found in other inorganic crystals. Obviously, there are large uncertainties in the data reported by different groups using different methods measured at different times for some of these $\mathrm{CSH}$ crystals. Hence, accurate theoretical computations based on density functional theory can provides the much needed consistency for CSH crystal structures based upon which reliable properties can be evaluated.

Bond order calculation in CSH. The bond order (BO) between each pair of atoms represents the quantitative measure of the bond stiffness and strength ${ }^{22}$. They are important in revealing the origin of internal cohesion in the CSH crystals. We obtain the $\mathrm{BO}$ values between every pair of atoms in the crystal using the first-principles orthogonalized linear combination of atomic orbitals (OLCAO) method $^{23}$ (see Method Section and Supplementary Information for details). Figure 2 shows the $\mathrm{BO}$ vs bond length (BL) distributions for four representative $\mathrm{CSH}$ crystals from each group (a: alite, b: afwillite, c: suolunite, and d: jennite). Different bond types in each crystals

\begin{tabular}{|c|c|c|c|c|c|c|}
\hline a. 1 & $5.565(-1.1)$ & $6.8(-0.6)$ & $9.363(-0.4)$ & $90(0)$ & $94.70(-0.6)$ & $90(0)$ \\
\hline a. 3 & $3.614(-0.6)$ & $3.614(-0.6)$ & $4.958(-1.1)$ & $90.18(-0.2)$ & $89.82(0.2)$ & $120.025(0)$ \\
\hline b. 1 & $16.57(-1.5)$ & $5.719(-1.4)$ & $11.764(-0.7)$ & $87.59(2.8)$ & $127.06(-0.5)$ & $93.65(-4.1)$ \\
\hline b. 2 & $9.535(-0.5)$ & $9.228(-0.5)$ & $10.777(-1)$ & $90(0)$ & $90(0)$ & $90(0)$ \\
\hline c. 1 & $7.052(-1.4)$ & $9.548(-0.7)$ & $6.883(-1)$ & $108.78(-0.1)$ & $95.73(-0.9)$ & $95.34(0.6)$ \\
\hline c. 2 & $19.988(-1.1)$ & $6.036(-0.8)$ & $11.239(-1.1)$ & $90(0)$ & $90(0)$ & $90(0)$ \\
\hline c. 3 & $11.527(-0.9)$ & $5.13(-0.8)$ & $22.204(-1.2)$ & $90(0)$ & $90(0)$ & $90(0)$ \\
\hline c. 4 & $20.638(-0.8)$ & $15.607(-0.9)$ & $6.898(-0.9)$ & $90(0)$ & $97.17(0.5)$ & $89.87(0.1)$ \\
\hline c. 5 & $10.085(-0.5)$ & $10.084(-0.5)$ & $7.538(-0.5)$ & $90(0)$ & $90(0)$ & $120(0)$ \\
\hline d. 1 & $7.665(-1)$ & $9.797(0)$ & $7.418(-1.1)$ & $110.94(0.7)$ & $103.59(-0.1)$ & $86.69(-0.2)$ \\
\hline d.7 & $10.423(-1)$ & $7.367(-0.1)$ & $7.159(-1.7)$ & $90.07(-0.1)$ & $106.48(-0.1)$ & $89.98(0)$ \\
\hline d. 8 & $10.732(-1.5)$ & $7.349(-1.2)$ & $10.784(1.3)$ & $103.22(-1.9)$ & $94.78(2.3)$ & $110.24(-0.5)$ \\
\hline
\end{tabular}


(Ca-O, Si-O, H-O-H, $-(\mathrm{O}-\mathrm{H}), \mathrm{H}_{2} \mathrm{O} \cdots \mathrm{H}_{2} \mathrm{O}$ and $-(\mathrm{O} \cdots \mathrm{H})$ ) are as indicated. As evident, there are two types of $\mathrm{OH}$ bonds. The strong covalently bonded ones and the weaker HBs. Further, there are two types of covalent $\mathrm{O}-\mathrm{H}$ bonds: those within the water molecules $(\mathrm{H}-$ $\mathrm{O}-\mathrm{H}$ ) which do not contribute to internal cohesion, and those in the hydroxyl ions $-(\mathrm{O}-\mathrm{H})$, which attach to silicates $(\mathrm{Si}-\mathrm{OH})$ or $\mathrm{Ca}$ ions $(\mathrm{Ca}-\mathrm{OH})$. The HBs also have two categories: HBs between water molecules $\left(\mathrm{H}_{2} \mathrm{O} \cdots \mathrm{H}_{2} \mathrm{O}\right.$, shown as inset of Figure 2 for b.1), and all other types of $\mathrm{HBs}$ that are not exclusively between two isolated water molecules. For example, the $\mathrm{HBs}$, such as $\mathrm{Si}-(\mathrm{O} \cdots \mathrm{H}) \mathrm{O}-\mathrm{Si}$ and $\mathrm{H}_{2}(\mathrm{O} \cdots \mathrm{H}) \mathrm{O}-\mathrm{Si}$, that are particularly significant are shown as inset of Figure 2 for c. 2 and d.8, respectively. As an overall measure of the crystal cohesion, we define the total $\mathrm{BO}$ (TBO) to be the sum of individual $\mathrm{BO}$ values and the total bond order density (TBOD) as the TBO normalized by the crystal volume.

The pie charts in Figure 2 give the percentage contribution from different types of bonds to the TBO. (Note: We have ignored the BO contribution from covalent $\mathrm{O}-\mathrm{H}$ bonds from water molecule in calculating the percentage contributions since they do not directly contribute to the overall cohesion.) Figure 2 gives an overall representative picture of the distribution and relative strengths of different bond species in CSH crystals. The broad, almost continuous distribution of $\mathrm{Si}-\mathrm{O} \mathrm{BO}$ values within a fairly narrow range of $\mathrm{Si}-\mathrm{O}$ BL clearly indicates that the bond stiffness/strength, not the bond length is the primary reason as to why the spectral features associated with $\mathrm{Si}-\mathrm{O}$ bond often appear as broad peaks ${ }^{24}$. It is also remarkable that $\mathrm{HBs}$ cover a wide range of $\mathrm{BO}$ and $\mathrm{BL}$ in these materials and have a significant contribution to the TBO. The effects of HBs have been widely speculated in experimental spectroscopic techniques but never clearly explained. In the following, we describe the BO distributions for crystals in each group in relation to their structural characterization, showing the complex picture of different BO contributions to the crystal cohesion.

Bond order distribution for each group. Figure S3 displays the plots of $\mathrm{BO}$ versus $\mathrm{BL}$ and the percentage contribution from different types of bonds to the TBO for all 20 crystals. In group a, Belite (a.1) and Alite (a.2), contain only $\mathbf{Q}^{0}$ silicates. The $\mathrm{Si}-\mathrm{O}$ bonds have a narrow BO distribution centered at $\sim 0.27$ with $\mathrm{BL}$ centered at $\sim 1.65 \AA$. The much weaker $\mathrm{Ca}-\mathrm{O}$ bonds exhibit a larger $\mathrm{BO}$ and $\mathrm{BL}$ dispersion ranging from 0.02 to 0.10 and $2.25 \AA$ to $3.50 \AA$, respectively. The $\mathrm{Ca}-\mathrm{O}$ bonds in Belite have almost evenly spread $\mathrm{BO}$ and $\mathrm{BL}$, while those in Alite show some clustering between $2.27 \AA$ to $2.55 \AA$. These $\mathrm{BO}$ and $\mathrm{BL}$ distributions clearly explain why the strong and
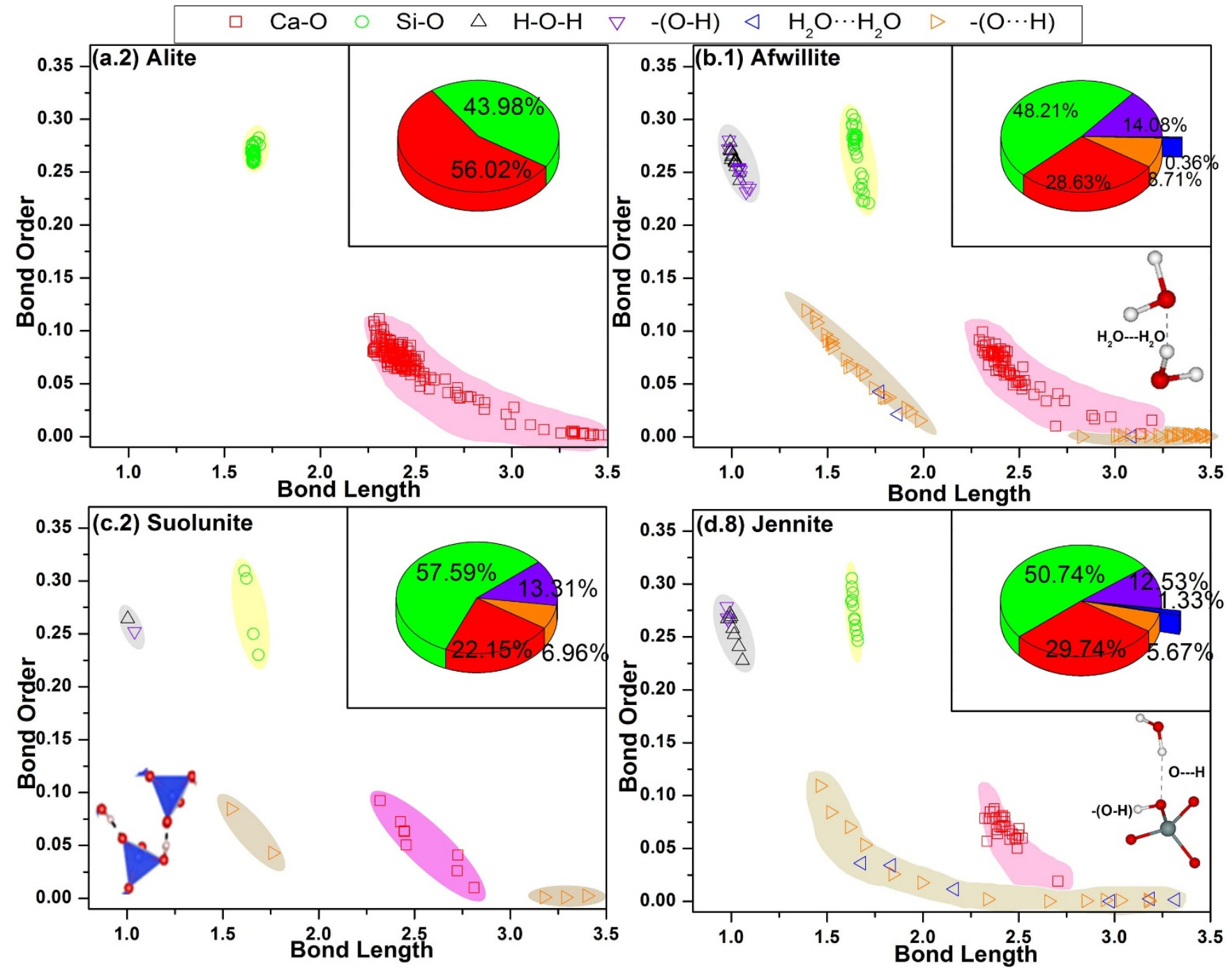

Figure $2 \mid$ Distribution of bond order vs. bond length in four representative crystals. (a.2) Allite; (b.1) Alwillite; (c.2) Suolunite; and (d.8) Jennite. Symbols for different types of bonds are shown at the top, “- “ for covalent bond, “..." for hydrogen bond. The pie chart in the inset shows the percentage of different types of bonding: red, Ca-O; green, Si-O; violet covalent - $(\mathrm{O}-\mathrm{H})$; orange, other $\mathrm{HB}$; blue, HB between $\mathrm{H} 2 \mathrm{O}$. Lower insets show different types of hydrogen bonds. 
considerably sharper $\mathrm{Si}-\mathrm{O}$ peaks dominate the experimental vibrational spectroscopic analyses of these minerals in contrast to the broad and weaker peaks associated with $\mathrm{Ca}-\mathrm{O}$ bonds ${ }^{25,26}$. It is noteworthy that in Portlandite (a.3) the BO for Ca-O bonds $(0.223$ with a $\mathrm{BL}$ of $2.381 \AA$ ) is much stronger than the $\mathrm{Ca}-\mathrm{O}$ bonds in the above CS crystals and all other CSH crystals.

The group $\boldsymbol{b}$ crystals contain the $\mathbf{Q}^{0}$ silicates as well as covalent $\mathrm{O}$ $\mathrm{H}$ bonds and HBs. In this group, only Afwillite contains water molecules. These crystals have a broadly dispersed $\mathrm{Si}-\mathrm{O} \mathrm{BO}$ although with well-defined narrowly ranged $\mathrm{BL}$. The lower Si-O BO is generally associated with the $\mathrm{Si}-\mathrm{OH}$ (hydroxyl) bond, while the higher $\mathrm{BO}$ with $\mathrm{Si}-\mathrm{O}$ bonds within the tetrahedral unit. The Ca-O BO in group $\boldsymbol{b}$ crystals is significantly smaller and shows a narrow distribution over a wide range of BL. Further, the covalent $\mathrm{O}-\mathrm{H}$ bonds and $\mathrm{HBs}$ exhibit a wide range of $\mathrm{BO}$ and $\mathrm{BL}$ as expected. For all the crystals in this group, the contributions from $\mathrm{Ca}-\mathrm{O}, \mathrm{O}-\mathrm{H}$ bonds and $\mathrm{HB}$ s to the TBO are almost $50 \%$ or more. For example, in Afwillite (b1), the contributions to the TBO are as follows: $\mathrm{Si}-\mathrm{O}$ (48.2\%), Ca-O (28.6\%), O-H $(14.1 \%)$ and $\mathrm{O} \cdots \mathrm{H}(8.7 \%)$. Here the covalent $\mathrm{O}-\mathrm{H}$ bonds are connected to the silicate tetrahedral units $\left[\mathrm{HSiO}_{4}\right]^{-3}$ through $\mathrm{HBs}$ whose BLs varies between 1.38-1.98 $\AA$ with significant $\mathrm{BO}$ values. Therefore, the $-\mathrm{O} \cdots \mathrm{H}$ contribution in Afwillite is the highest $(8.71 \%)$ in this group. Rastsvetaeva et $\mathrm{al}^{24}$, in their IR spectrum analyses of Afwillite ${ }^{27}$, have assigned the characteristic vibrational modes of the so-called zundel cation $\left(\mathrm{H}_{2}-\mathrm{O} \cdots \mathrm{H} \cdots \mathrm{OH}_{2}\right)^{+}$, or $\left(\mathrm{H}_{5} \mathrm{O}_{2}{ }^{+}\right)^{28}$. However, in our $\mathrm{BO}$ analysis, we do not observe this zundel cation structure. Instead, we find two HBs connecting water molecules with close proximity on either side of $\mathrm{CaO}$ layer (parallel to b-c plane) as shown in Figure 1 (b.1). Clearly, the interpretation of the IR spectrum could have immensely benefitted from the relaxed structure and $\mathrm{BO}$ calculations. In contrast, for $\alpha$-C2SH (b.2), which contains both $\mathrm{Si}-\mathrm{OH}$ and $\mathrm{Ca}-\mathrm{OH}$, the $\mathrm{HB}$ related to $\mathrm{Si}-\mathrm{OH}$ is relatively stronger $(\mathrm{BO}=0.066)$ compared to those linked to $\mathrm{Ca}-\mathrm{OH}$ $(\mathrm{BO}=0.014)$. The stronger HBs in $\alpha-\mathrm{C} 2 \mathrm{SH}$ (b.2) can be attributed to the fact that this crystal is formed under the high temperature hydrothermal conditions and is stable up to very high temperature ${ }^{20}$. The other two crystals in group $\boldsymbol{b}$, Dellaite (b.3) and Ca-Chondrodite (b.4), show negligible contribution from the HBs because of the larger $\mathrm{O} \cdots \mathrm{H}$ separations. The $\mathrm{Si}-\mathrm{O}$ bond, the $\mathrm{Ca}-\mathrm{O}$ bond and the covalent $\mathrm{O}-\mathrm{H}$ bond make primary contribution to TBO in these two crystals.

Crystals in the group $c$ have an even wider distribution of Si-O BO values than group $\boldsymbol{b}$, ranging from 0.18 to 0.35 . The contribution from $\mathrm{Si}-\mathrm{O}$ bonds to TBO is generall, larger than in group $\boldsymbol{b}$ whereas contribution from $\mathrm{HBs}$ is comparatively less. In this group, Kilchoanite (c.3) is a naturally occurring anhydrous crystal ${ }^{29}$ with two types of silicate chains $\left(\mathbf{Q}^{0}\right.$ and $\left.\mathbf{Q}^{2}\right)$ coexisting ${ }^{30}$. For crystals other than Kilchoanite (c.3), the O-H covalent bonds are clustered in a very narrow range similar to group $\boldsymbol{a}$ and $\boldsymbol{b}$ and the HBs follow the similar range as in group $\boldsymbol{b}(1.5 \AA-1.80 \AA)$. Although the group $c$ crystals share the Strunz classification, they exhibit wide differences in structural and bonding characteristics. For example, the Killalaite (c.4) is a complex non-stoichiometric crystal with partial occupancy. It is composed of $Q^{1}$ silicate chains via a corner sharing $\mathrm{O}$ with the $\mathrm{Si}$ $\mathrm{O}-\mathrm{Si}$ angle of $124.8^{\circ}$. This crystal contains both $\mathrm{Si}-\mathrm{OH}$ and $\mathrm{Ca}-\mathrm{OH}$ units. The Si-O BO distribution is very broad (0.195-0.322) over a relatively narrow range in $\mathrm{BL}(1.60-1.72 \AA)$. The HBs have relatively high BOs $(0.045-0.102)$ with a BL in the range of (1.50-1.74 $\AA$ ). The highest $\mathrm{BO}$ of $\mathrm{HBs}$ are $\mathrm{Si}-\mathrm{OH} \cdots \mathrm{O}$ and $\mathrm{Ca}-\mathrm{OH} \cdots \mathrm{O}$. The percentage contribution to $\mathrm{TBO}$ from $\mathrm{Si}-\mathrm{O}, \mathrm{Ca}-\mathrm{O}, \mathrm{O}-\mathrm{H}$, and $\mathrm{HBs}$ are $54.3 \%$, $34.33 \%, 10.03 \%$ and $1.33 \%$ respectively. On the other hand, Jaffeite (c.5) has a completely different structure and bonding with 12 covalent $\mathrm{OH}$ bonds in the form of $\mathrm{Ca}-\mathrm{OH}$ but virtually no HBs. All the hydroxyl groups connected to $\mathrm{Ca}$ are directed away from the disilicate chains. Clearly, Jaffeite (c.5) is an outlier in terms of BO contributions compared to the other crystals in $c$. It has the lowest $\mathrm{Si}-\mathrm{O}$ contribution (34.1\%) among the 20 crystals considered, hence the lowest TBOD. Of all the crystals in group c, Suolunite (c.2) is the most conspicuous. It contains primarily $\mathbf{Q}^{1} \mathrm{Si}$-tetrahedra, as opposed to others that have both $\mathbf{Q}^{0}$ and $\mathbf{Q}^{2}$. Suolunite (c.2) is the only crystal in this group that contains water molecules, and is the only naturally occurring CSH crystal to have a $\mathrm{CaO}: \mathrm{SiO}_{2}: \mathrm{H}_{2} \mathrm{O}$ ratio of $1: 1: 1^{31}$. All water molecules in Suolunite (c.2) have a consistent covalent bond length of $1.0033 \AA$. Since these water molecules are far apart so the $\mathrm{H}_{2} \mathrm{O} \cdots \mathrm{H}$ HBs are absent. All the other HBs have short BL (1.5-1.76 $\AA$ ) and relatively strong BO (0.042-0.084). Both $\mathbf{Q}^{0}$ and $\mathbf{Q}^{1}$ type silicates units in Suolunite (c.2) have one covalent O-H bond which also make $\mathrm{HBs}$ with the nearby $\mathrm{O}$ of the silicate chain with a BL (1.543 $\AA$ ) and $\mathrm{BO}(0.085)$. The rest of $\mathrm{HBs}$ are from water molecule to the $\mathrm{O}$ of the adjacent silicate chains. It is interesting to note that $-\mathrm{O} \cdots \mathrm{H}$ has twice as large $\mathrm{BO}$ value than the HBs from water molecules. Suolunite (c.2) and Rosenhahnite (c.1) have same C/S ratio but the latter has no water molecules. Hence, the HBs contributions to the TBO are significantly small. We will return to more focused discussion on Suolunite later.

In group $\boldsymbol{d}$ crystals, the contribution of $\mathrm{Si}-\mathrm{O}$ bonds in the infinite silicate chain structures dominates the TBO. However, the Si-O contribution to the $\mathrm{TBO}$ generally decreases as the $\mathrm{C} / \mathrm{S}$ ratio increases. In addition, the layer separation, $\mathrm{H}_{2} \mathrm{O} / \mathrm{OH}$ content and crystal symmetry also affect the $\mathrm{BO}$ contributions. The CSH crystals in this group are amongst the most hydrated, except Wollastonite (d.5) which is an anhydrous precursor phase with $71.8 \%$ Si-O bonds and $28.2 \% \mathrm{Ca}-\mathrm{O}$ bonds $^{32}$. The Si-O BO values of this group exhibit dispersion primarily due to small distortions from the ideal tetrahedral unit and the hydroxyl ions connecting the silicate chains and $\mathrm{Ca}$. Two types of silicate chains are observed: (1) the single silicate chain as those found in d.1, d.3, d.4, d.5, d.7 and d.8; and (2) the double silicate chain in which 2 silicate chains from either side of the interlayer are connected via a bridging-O as in $\mathrm{d} .2$ and d. $6^{20}$. The presence of silicate chains leads to a layered structure of these minerals as well as high contribution of $\mathrm{Si}-\mathrm{O}$ to the TBO. The interlayer separations between layers range from $4.95 \AA$ in Foshagite (d.7) to $14 \AA$ in T14 (d.3). The layered structure formed by these chains has a significant effect on the mechanical anisotropy of these crystals. Typically, water molecules and Ca reside in interlayer cavities and govern the interlayer cohesion ${ }^{14}$. For example in Nekoite (d.1), the interlayer (of separation 7.6 ) contains all $\mathrm{Ca}$ and $\mathrm{H}_{2} \mathrm{O}$. In this case, the $\mathrm{Ca}-\mathrm{O}$ bond contributes $15.24 \%$ and the $\mathrm{HBs}$ contribute $5.21 \%$ to the interlayer cohesion. In contrast, the $\mathrm{T} 11$ (d.2) has $\mathrm{H}_{2} \mathrm{O}$ in the interlayer but no Ca, thus HBs contribute 4.04\%, whereas in T14 (d.3) and T9 (d.4), which has both $\mathrm{Ca}$ and $\mathrm{H}_{2} \mathrm{O}$, the $\mathrm{Ca}-\mathrm{O}$ bond contributes $4.07 \%$ and $4.86 \%$ and the HBs contribute $5.48 \%$ and $3.17 \%$, respectively to the to the interlayer cohesion. The difference in T14 (d.3) and T9 (d.4) is attributed to the lack of double silicate chains in former and the presence in later. Further, Xonotlite (d.6) and Foshagite (d.7) have unusually low contribution of HBs since these crystals contain only hydroxyl ions. In group $\boldsymbol{d}$, Jennite (d.8), which has been widely considered as part of the C-S-H phase in hydrated cement, has the highest contribution of HBs (6.99\%) to the interlayer cohesion.

\section{Discussion and conclusion}

We have presented the calculated electronic structure of $15 \mathrm{CSH}$ crystals, 4 anhydrous precursor crystals and portlandite crystal. Band structures show all crystals are large band gap insulators. The BO topology and their contribution to the overall cohesion via TBO are analyzed. We found no specific rule common to all crystals but observed some trends for each group separately. The BO, TBO and TBOD of the various crystals clearly shows (1) the diverse degree of contributions of the various bond types to the overall crystal cohesion, (2) the variety of HBs, (3) the BO dispersion within each bond types in $\mathrm{CSH}$, and (4) the importance of accurate crystal structure whose volume determines the TBOD. The traditional criterion for 
classifying $\mathrm{CSH}$ crystals using $\mathrm{C} / \mathrm{S}$ ratio is rather rudimentary in view of these diversities. We propose the total bond order density (TBOD) as an accurate quantum mechanical metric to classify the CSH crystals that provides far deeper insight to cohesion and strength of these materials.

Figure 3(a) shows the plot of TBOD vs the C/S ratio showing an approximate inverse relationship. It is apparent that crystals with the same $\mathrm{C} / \mathrm{S}$ ratio can have very different TBOD. Since TBOD is more sensitive to the bonding and relevant to cohesion, its use for classifying CSH crystals is most appropriate. This plot also shows that the four groups discussed above do not cluster together in relation to the TBOD. Group $c$ has a wide range of $\mathrm{C} / \mathrm{S}$ as well as TBOD. However, group $\boldsymbol{d}$, believed to be closest to hydrated cement, has a narrow distribution in $\mathrm{C} / \mathrm{S}$ but a wider TBOD range. Roughly speaking, we can divide the 20 crystals into to 3 groups according to their TBOD high, medium and low. Crystals a.1, b.4 and c.5 belong to the low TBOD group mainly because of the high $\mathrm{C} / \mathrm{S}$ ratio. On the opposite end, b.1 from group $b, c .1$ and c. 2 from group $c$ and d.1, d2 and $\mathrm{d} 4$ from group $d$ belong to the high TBOD group. The rest of 10 crystals belong to the medium TBOD group which mostly from group $d$ crystals. The mixing of groups based upon Strunz classification and a weak correlation of TBOD with $\mathrm{C} / \mathrm{S}$ ratio signifies the complex interplay and tradeoffs between the covalent bonds and HBs in these $\mathrm{CSH}$ structures. Although, $\mathrm{Q}^{\mathrm{n}}$ chains in group $\boldsymbol{d}$ crystals tend to reduce $\mathrm{C} / \mathrm{S}$ ratio, interlayer hydroxyl, water and $\mathrm{HBs}$ drastically alter the TBOD by increasing the effective volume without modification to $\mathrm{C} / \mathrm{S}$ ratio. For example, crystal $\mathrm{T} 14$ (d.3) has a low $\mathrm{C} / \mathrm{S}$ ratio but still has medium TBOD. The reason is that there are two types of silicate. Both d.2 and d.4 structures have double silicate chains whereas d.3 has only single silicate chains, which affects the TBOD considerably.

Measured mechanical properties for CSH crystals are difficult to obtain due to various practical limitations such as challenges in synthesizing single crystals, fast reaction with atmospheric water etc. Thus, there are only a few of CSH/CS phases where the mechanical properties were obtained experimentally. For example, the clinker phases Belite (a.1) and Alite (a.2), which have well-defined stoichiometric structures, are reported to have experimentally measured Young's modulus (E) between 140-130 GPa and 147-135 GPa respectively ${ }^{33,34}$. Similar experiments reported the E for Portlandite (a.3) to be in the range 36-48 $\mathrm{GPa}^{34-38}$ depending on the porosity and a bulk modulus value of $39.65 \mathrm{GPa}^{39}$. The bulk modulus of the crystal Toberomite $14 \AA$ (d.3) obtained recently using high pressure synchrotron X-ray diffraction to be $47 \mathrm{GPa}^{40}$ Using these limited experimental values, we find that the average of the measured $E$ values for a.1, a. 2 and a. 3 crystals increase as a function of TBOD and show a near linear fit. In addition, the measured bulk modulus found for the two crystals, i.e. a. 3 and d. 3 also increase with TBOD. At this stage it is premature to correlate experimental mechanical properties with calculated TBOD. We intend to study this further with the calculated mechanical properties to find possible trends of mechanical properties with the TBOD.

Since $\mathrm{Ca}, \mathrm{Si}$ and $\mathrm{H}$ are the primary components of the various bond types in $\mathrm{CSH}$, it is useful to examine the complex interactions amongst the bond types by investigating the TBOD as function of $\mathrm{Ca}: \mathrm{Si}: \mathrm{H}$ ratio. Figure 3(b) shows the color-coded ternary plot of TBOD map with respect to $\mathrm{Ca}: \mathrm{Si}: \mathrm{H}$ composition. The resultant contour plot gives a vivid illustration of the possible locations for phases with high TBOD which appear to concentrate in the region where $\mathrm{Ca}: \mathrm{Si}: \mathrm{H}=0.25: 0.25: 0.5$. We note that Soulunite (c.2) with $\mathrm{C} / \mathrm{S}$ ratio equal to 1 has the highest TBOD out of the 20 crystals investigated and according to our criterion, would be closest to the ideal composition and structure. The electronic structure and bonding of Soulunite has not been investigated until now. In the SI, we have further discussed its electronic structure with the presentation of its total density of states (DOS) and partial density of states (PDOS) resolved in accordance with the atoms involved in different types of bonding. The narrow green and yellow areas in Figure 3(b) represent the medium TBOD. The wider blue regions represent the low TBOD regions.

In C-S-H (cement hydrates), it is known that infinite chain structures are absent ${ }^{1}$. On the other hand, it is widely believed that mixtures of tobermorite (d.2-d.4) and jennite (d.8) form the template or backbone of the C-S-H disordered structure. However, the TBOD and the $\mathrm{BO}$ calculations show that $\mathrm{C}-\mathrm{S}-\mathrm{H}$ structures in hydrated cements cannot simply be surmised as mixtures of CSH crystals or even some disordered form of these mixtures. In this regard, the following three aspects become clear: (1) the significant contribution of HBs and their role in affecting the BO of each bond type, (2) the significant dispersion in $\mathrm{BO}$ of the strong $\mathrm{Si}-\mathrm{O}$ bonds with small $\mathrm{BL}$ variation, and (3) the variation in the $\mathrm{BO}$ of relatively weaker $\mathrm{Ca}-\mathrm{O}$ bond. Since the interplay between different components from the structures cannot be explicitly quantified by known experiments ${ }^{7}$, detailed electronic structure and bonding information are necessary to interpret experimental findings, especially in spectroscopic studies such as in Raman, IR and NMR measurements ${ }^{18,24,41}$. The implication is that attempts to use vibrational spectroscopy, such as IR or Raman, for structural interpretation have to be viewed carefully with special attention paid to small features and nuances, such as peak width and asymmetry.

From the viewpoint of mechanical properties, which are highly important for this material class, we note that silicates with strong covalent bonds are expected to contribute significantly to the overall crystal stiffness under small deformation. However, hydrated crystals with a low degree of polymerization may have considerable contributions to the materials stiffness via HBs in the HB network. Bond order analysis on crystals with finite silicate chains shows that $\mathrm{H}_{2} \mathrm{O}, \mathrm{OH}$ and $\mathrm{HB}$ network all play a key role in the cohesion of crystals. More significantly, the $\mathrm{Si}-\mathrm{O}$ covalent bonds are unlikely to be the main source of crystal strength as often reported in the literature $^{42}$. In multi-component crystals that include ionic, covalent and hydrogen bonds, the nonlinearity of mechanical response, the failure and the fracture behavior is often controlled by the weaker ionic or hydrogen bonds. Indeed, in these complex material systems, different atomic-scale deformation mechanisms trigger and persist under different loading paths ${ }^{43}$. For the CSH crystals as well as the more disordered $\mathrm{C}-\mathrm{S}-\mathrm{H}$, both the silicate polymerization and the $\mathrm{BO}$ of various bond types will affect the deformation mechanisms. In this respect, highly polymerized structures, such as Tobermorite and Jennite, are likely to have highly anisotropic mechanical response with stiff response along the silicate chains complimented by planes of weaknesses. Suolunite, which has the highest TBOD, a high contribution of HBs and a low degree of polymerization of silicates, is likely to have a comparably high stiffness and absence of weak planes.

In conclusion, we have presented a compelling argument for using a quantum mechanical metric, the $\mathrm{BO}$ and the TBOD as the most important criterion to assess the crystalline cohesion in CSH crystals. This metric encompasses effects of both the geometric factors and the interatomic interactions. Therefore, the BO and TBOD provide a much more in depth measure for the overall behavior of this diverse class of materials. The TBOD should certainly replace the longstanding and traditionally used $\mathrm{C} / \mathrm{S}$ ratio in classifying cement materials. Of the $15 \mathrm{CSH}$ crystals studied, the inconspicuous crystal Suolunite turns out to have the highest TBOD, and the ideal composition, structure and bonding characteristics for cement hydrate. This finding should serve as an impetus for cement scientists engaged in molecular scale engineering of hydraulic cements. Synthesis of new anhydrous precursors or hydration pathways that lead to Suolunite type hydration products could yield robust infrastructural materials. Furthermore, the results of this study make it amply clear that the understanding of electronic structure and inter-atomic bonding characteristics is essential for the interpretation of experimental spectroscopic data as well as for the development of realistic 


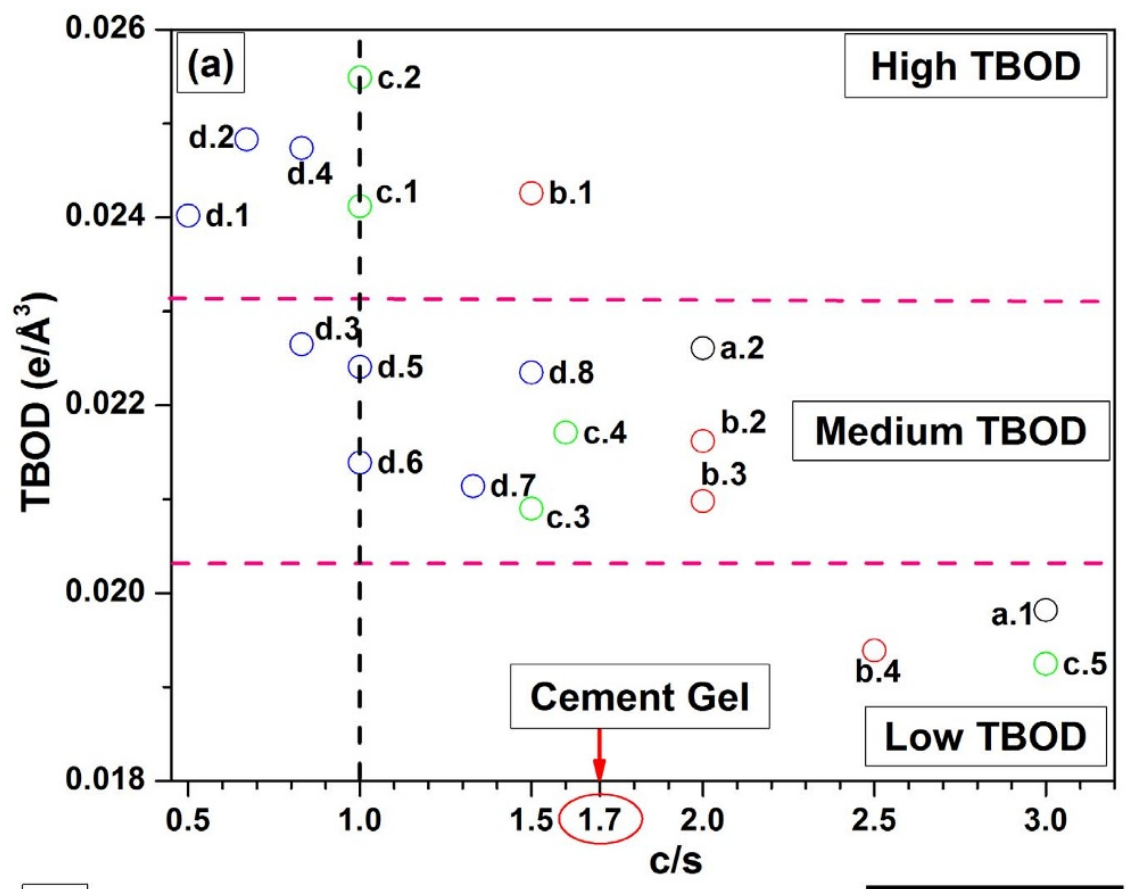

(b)

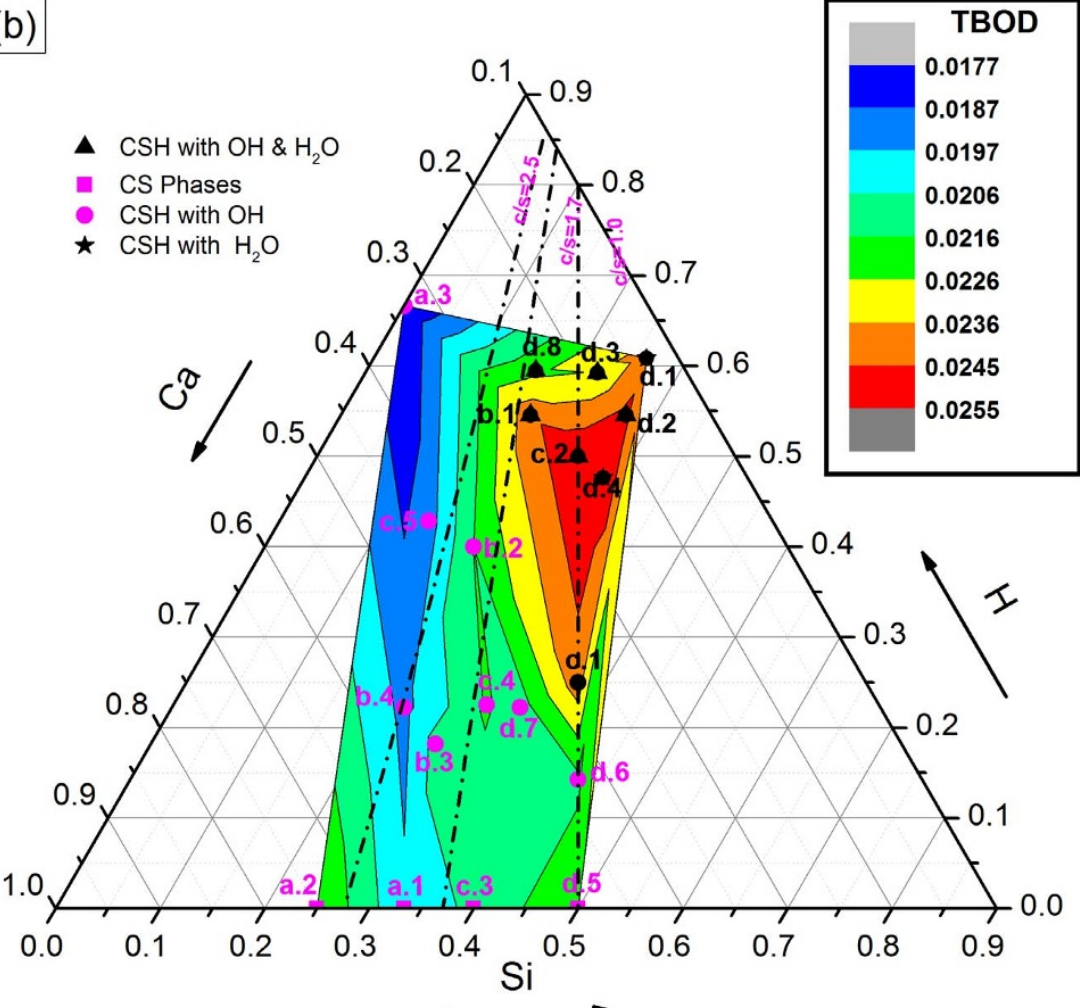

Figure $3 \mid$ (a) Total bond order density vs. C/S ratio in 19 crystals. (Portlandite (a.3) was excluded since it has no Si atoms.); (b) TBOD distribution map vs. the composition of $\mathrm{Ca}: \mathrm{Si}: \mathrm{H}$. The color bar labels the values for the TBOD. The dot-dash lines indicate specific $\mathrm{C} / \mathrm{S}$ ratios.

structural models of disordered cement hydrates. The approach used in this study has application to a broad class of complex material systems that incorporate covalent, ionic and hydrogen bonds, such as the apatite mineral class relevant to biological systems.

\section{Methods}

Crystal structural relaxation. For structural relaxation of the CSH crystals, we used the Vienna ab initio simulation package (VASP ${ }^{44,45}$. The technical details are briefly outlined as follows: (1) the PAW-PBE potentials ${ }^{46,47}$ with the generalized gradient approximation (GGA) is used. (2) The electronic convergence criterion is set at
$10^{-6} \mathrm{eV}$. (3) The force convergence criterion is set at $10^{-5} \mathrm{eV} / \AA$ for ionic relaxation. (4) A high energy cutoff of $500 \mathrm{eV}$ is adopted and (5) A sufficiently large number of $\boldsymbol{k}$-point-meshes ranging from 2 to $85 \mathrm{k}$-points are used depending on the actual crystal structure and size.

Bond order density. For electronic structure and bonding analysis, we used the orthogonalized linear combination of atomic orbitals (OLCAO) method $\mathrm{d}^{23,48}$ based on the VASP-relaxed crystal structures. The bond order values for each crystal are calculated using Mullikan population analysis ${ }^{49}$. A more detailed description of the $\mathrm{BO}$ calculation is included in the SI. The TBOD is obtained as the cumulative total $\mathrm{BO}$ from all unique bond pairs divided by the cell volume. The percentage contribution of $\mathrm{BO}$ does not include the covenant $\mathrm{O}-\mathrm{H}$ bonds of the water 
molecules since they do not directly contribute to the internal cohesion of the crystal.

1. Richardson, I. G. The calcium silicate hydrates. Cement Concrete Res 38, 137-158 (2008).

2. Bernal, J. D. The structures of cement hydration compounds. Paper presented at Proc. 3rd Int. Symp. Chem. Cem., London: Cement and Concrete Association. pp. 216-236 (1952).

3. Taylor, H. \& Howison, J. Relationships between calcium silicates and clay minerals. Clay Minerals Bull 3, 98-111 (1956).

4. Glasser, F. P., Lachowski, E. E. \& Macphee, D. E. Compositional Model for Calcium Silicate Hydrate (C-S-H) Gels, Their Solubilities, and Free Energies of Formation. J Am Ceram Soc 70, 481-485 (1987).

5. Taylor, H. F. W. \& Roy, D. M. Structure and Composition of Hydrates at 7th International Congress on the Chemistry of Cement: Principal Reports, Paris. Editions Septima, PII 2/1-13 (1980).

6. Dolado, J. S. \& Van Breugel, K. Recent advances in modeling for cementitious materials. Cement Concrete Res 41, 711-726 (2011).

7. Cong, X. \& Kirkpatrick, R. J. ${ }^{29}$ Si MAS NMR study of the structure of calcium silicate hydrate. Adv Cem Based Mater 3, 144-156 (1996).

8. Pellenq, R. J.-M. et al. A realistic molecular model of cement hydrates. P Natl Acad Sci USA 106, 16102-16107; DOI:10.1073/pnas.0902180106 (2009).

9. Taylor, H. F. W. Proposed Structure for Calcium Silicate Hydrate Gel. J Am Ceram Soc 69, 464-467 (1986).

10. Taylor, H. F. W. Tobermorite, jennite, and cement gel. Zeitschrift für Kristallographie 202, 41 (1992).

11. Pellenq, R.-M., Caillol, J. \& Delville, A. Electrostatic attraction between two charged surfaces: a (N, V, T) Monte Carlo simulation. J Phys Chem B 101, 8584-8594 (1997).

12. Dolado, J. S., Griebel, M. \& Hamaekers, J. A molecular dynamic study of cementitious calcium silicate hydrate (C-S-H) gels. J Am Ceram Soc 90, 3938-3942 (2007).

13. Richardson, I. G. The nature of C-S-H in hardened cements. Cement Concrete Res 29, 1131-1147 (1999).

14. Dharmawardhana, C. C., Misra, A., Aryal, S., Rulis, P. \& Ching, W. Y. Role of interatomic bonding in the mechanical anisotropy and interlayer cohesion of CSH crystals. Cement Concrete Res 52, 123-130 (2013).

15. Strunz, H. Mineralogische Tabellen. (Akad. Verl.-Ges. Geest u. Portig, 1982).

16. Jennings, $\mathrm{H}$. M. A model for the microstructure of calcium silicate hydrate in cement paste. Cement Concrete Res 30, 101-116 (2000).

17. Tennis, P. D. \& Jennings, H. M. A model for two types of calcium silicate hydrate in the microstructure of Portland cement pastes. Cement Concrete Res 30, 855-863 (2000).

18. Cong, X. \& James Kirkpatrick, R. ${ }^{17} \mathrm{O}$ and ${ }^{29} \mathrm{Si}$ MAS NMR study of $\beta-\mathrm{C} 2 \mathrm{~S}$ hydration and the structure of calcium-silicate hydrates. Cement Concrete Res $\mathbf{2 3}$, 1065-1077 (1993).

19. Thomas, S., Meise-Gresch, K., Müller-Warmuth, W. \& Odler, I. MAS NMR Studies of Partially Carbonated Portland Cement and Tricalcium Silicate Pastes. I Am Ceram Soc 76, 1998-2004 (1993).

20. Garbev, K., Black, L., Beuchle, G. \& Stemmermann, P. Inorganic polymers in cement based materials. Wasser-und Geotechnologie 1, 19-31 (2002).

21. De la Torre, Á. G., De Vera, R. N., Cuberos, A. J. M. \& Aranda, M. A. G. Crystal structure of low magnesium-content alite: Application to Rietveld quantitative phase analysis. Cement Concrete Res 38, 1261-1269 (2008).

22. Haslingerová, I. Estimation of bond energies from mulliken overlap populations. Czech J Phys 27, 1389-1393 (1977).

23. Ching, W.-Y. \& Rulis, P. Electronic Structure Methods for Complex Materials: The orthogonalized linear combination of atomic orbitals 24 (Oxford University Press, USA, 2012).

24. Rastsvetaeva, R. K., Chukanov, N. V. \& Zadov, A. E. Refined structure of afwillite from the northern Baikal region. Crystallogr. Rep. 54, 418-422 (2009).

25. Richardson, I., Skibsted, J., Black, L. \& Kirkpatrick, R. J. Characterisation of cement hydrate phases by TEM, NMR and Raman spectroscopy. Adv Cem Res 22, 233-248 (2010).

26. Doyle, S. G. Construction and Building: Design, Materials, and Techniques. (Nova Science Publishers, Incorporated, 2010).

27. Malik, K. M. A. \& Jeffery, J. W. A re-investigation of the structure of afwillite. Acta Crystallogr B 32, 475-480 (1976).

28. Kim, J., Schmitt, U. W., Gruetzmacher, J. A., Voth, G. A. \& Scherer, N. E. The vibrational spectrum of the hydrated proton: Comparison of experiment, simulation, and normal mode analysis. J Chem Phys 116, 737-746 (2002).

29. Agrell, S. O. \& Gay, P. Kilchoanite, a polymorph of rankinite. Nature 189, 743-743 (1961).

30. Roy, D. M., Gard, J. A., Nicol, A. W. \& Taylor, H. F. W. New Data for the Calcium Silicate, 'Phase Z'. Nature 188, 1187-1188 (1960).
31. Zhesheng, M., Nicheng, S., Guodong, M. \& Libing, L. Crystal structure refinement of suolunite and its significance to the cement techniques. Chin. Sci. Bull. 44, 2125-2130 (1999).

32. Ito, T.-i., Sadanaga, R., Takéuchi, Y. \& Tokonami, M. The existence of partial mirrors in wollastonite. P Jpn Acad Sci 45, 913-918 (1969).

33. Velez, K., Maximilien, S., Damidot, D., Fantozzi, G. \& Sorrentino, F. Determination by nanoindentation of elastic modulus and hardness of pure constituents of Portland cement clinker. Cement Concrete Res 31, 555-561 (2001).

34. Ulm, F., Bazant, Z. \& Wittman, F. Creep, Shrinkage, and Durability Mechanics of Concrete and Other Quasi-Brittle Materials. [197-203] (Elsevier, Amsterdam, 2001).

35. Constantinides, G. \& Ulm, F.-J. The nanogranular nature of C-S-H.J Mech Phys Solids 55, 64-90 (2007)

36. Beaudoin, J. J. Comparison of mechanical properties of compacted calcium hydroxide and portland cement paste systems. Cement Concrete Res 13, 319-324 (1983).

37. Wittmann, F. H. Estimation of the modulus of elasticity of calcium hydroxide. Cement Concrete Res 16, 971-972 (1986).

38. Monteiro, P. J. M. \& Chang, C. T. The elastic moduli of calcium hydroxide. Cement Concrete Res 25, 1605-1995 (1995).

39. Manzano, H., Dolado, J. S. \& Ayuela, A. Elastic properties of the main species present in Portland cement pastes. Acta Mater 57, 1666-1674 (2009).

40. Oh, J. E., Clark, S. M., Wenk, H.-R. \& Monteiro, P. J. M. Experimental determination of bulk modulus of $14 \AA$ tobermorite using high pressure synchrotron X-ray diffraction. Cement Concrete Res 42, 397-403 (2012).

41. Cong, X. \& Kirkpatrick, R. J. ${ }^{29} \mathrm{Si}$ and ${ }^{17} \mathrm{O}$ NMR investigation of the structure of some crystalline calcium silicate hydrates. Adv Cem Based Mater 3, 133-143 (1996).

42. Manzano, H. Atomistic Simulation Studies of the Cement Paste Components, $\mathrm{PhD}$ thesis, University of the Basque Country UPV/EHU, (15 $5^{\text {th }}$ July 2009).

43. Misra, A. \& Ching, W. Theoretical nonlinear response of complex single crystal under multi-axial tensile loading. Sci. Rep. 3, 1488; DOI:10.1038/ srep01488(2013).

44. Kresse, G. \& Hafner, J. Ab initio molecular dynamics for liquid metals. Phys Rev B 47, 558-561 (1993).

45. Kresse, G. \& Furthmüller, J. Efficiency of ab-initio total energy calculations for metals and semiconductors using a plane-wave basis set. Comp Mater Sci 6, 15-50 (1996).

46. Blöchl, P. E. Projector augmented-wave method. Phys Rev B 50, 17953-17979 (1994).

47. Perdew, J. P. Accurate Density Functional for the Energy: Real-Space Cutoff of the Gradient Expansion for the Exchange Hole. Phys Rev Lett 55, 1665-1668 (1985).

48. Ching, W. Y. Theoretical Studies of the Electronic Properties of Ceramic Materials. J Am Ceram Soc 73, 3135-3160 (1990).

49. Mulliken, R. S. Electronic Population Analysis on LCAO[Single Bond]MO Molecular Wave Functions. I. J Chem Phys 23, 1833-1840 (1955).

\section{Acknowledgments}

This research was supported by the NSF under grant CMMI-1068528. This research used the resources of NERSC supported by the Office of Science of DOE under the contract No. DE-AC03-76SF00098.

\section{Author contributions}

W.Y.C. and A.M. designed the research; C.C.D. and W.Y.C. performed the calculations; all authors analyzed the data; all authors contributed to the discussion and wrote the paper.

\section{Additional information}

Supplementary information accompanies this paper at http://www.nature.com/ scientificreports

Competing financial interests: The authors declare no competing financial interests. How to cite this article: Dharmawardhana, C.C., Misra, A. \& Ching, W.Y. Quantum Mechanical Metric for Internal Cohesion in Cement Crystals. Sci. Rep. 4, 7332; DOI:10.1038/srep07332 (2014).

This work is licensed under a Creative Commons Attribution-NonCommercialNoDerivs 4.0 International License. The images or other third party material in this article are included in the article's Creative Commons license, unless indicated otherwise in the credit line; if the material is not included under the Creative Commons license, users will need to obtain permission from the license holder in order to reproduce the material. To view a copy of this license, visit http:// creativecommons.org/licenses/by-nc-nd/4.0/ 